Cahiers de recherches médiévales

\title{
Une combinatoire du récit médiéval
}

Le château des destins croisés d'Italo Calvino

\section{Dominique Demartini}

\section{(2) OpenEdition \\ Journals}

Édition électronique

URL : https://journals.openedition.org/crm/2683

DOI : $10.4000 / \mathrm{crm} .2683$

ISSN : 1955-2424

Éditeur

Honoré Champion

\section{Édition imprimée}

Date de publication : 15 décembre 2007

Pagination : 233-249

ISSN : 1272-9752

Référence électronique

Dominique Demartini, « Une combinatoire du récit médiéval », Cahiers de recherches médiévales [En ligne], 14 | 2007, mis en ligne le 15 décembre 2010, consulté le 15 décembre 2022. URL : http:// journals.openedition.org/crm/2683; DOI : https://doi.org/10.4000/crm.2683 


\section{RM}

\section{Une combinatoire du récit médiéval : Le château des destins croisés d'Italo Calvino}

Dans le Château des destins croisés d'Italo Calvino, des chevaliers se retrouvent à une table d'hôtes et au moment de conter leur aventure, la parole leur fait défaut. Ils ont alors recours à un jeu de tarot pour figurer leur récit, et de carte en carte, chacun donne à deviner, à lire, sa propre histoire. Le tarot fonctionne ainsi, selon l'expression de Calvino, comme une «machine narrative combinatoire ${ }^{1}$. Si le tarot Visconti, avec ses enluminures du Quattrocento, sert de base à ce premier récit, le texte qui suit, L'auberge des destins croisés, reprend le même procédé, mais cette fois avec le tarot de Marseille. Dans la Note qui suit les deux textes, Calvino ajoute qu'il avait songé à un troisième, où figurerait, à la place du jeu de tarot, une bande dessinée. D'autres supports sont ainsi possibles, propres à illustrer la fabrique narrative comme machine combinatoire. Le plus souvent, ce rôle est dévolu au jeu d'échecs². En privilégiant comme support un jeu de tarot du Quattrocento, véritable répertoire iconographique médiéval, Calvino place donc la matière médiévale au cœur de son expérience narrative. On mettra en évidence ce que le tarot révèle du jeu poétique et, au sein de ce jeu, de la littérature médiévale. Cette dernière, ellemême souvent fragmentée, inachevée, privée d'auteurs, aux limites de la littérature, participe paradoxalement, à travers les cartes du jeu de tarot, à l'élaboration d'une théorie du récit, et apparaît comme un des rouages privilégiés de "la machine littérature » de Calvino ${ }^{3}$. La littérature médiévale offre non seulement un répertoire d'images, mais également une certaine conception et pratique du récit, qui met en œuvre, au fil des combinaisons narratives, le «dessous des cartes» du lecteur, sa part d'ombre et de lumière.

\section{La littérature médiévale comme jeu de cartes}

\section{traditionnel}

Le château-taverne ou comment Calvino joue avec le récit-cadre

Après avoir traversé la forêt, des chevaliers parviennent à un château. Réunis autour d'une table, l'un d'eux prend la parole pour raconter ses aventures. Tel est le cadre des deux nouvelles de Calvino : Le château des destins croisés et La taverne des destins croisés. ${ }^{4}$ C'est également celui de bon nombre de romans de Chrétien,

${ }^{1}$ I. Calvino, Le Château des destins croisés, Paris, Seuil, 1976, Note, p. 134.

${ }^{2}$ Cf. Échiquiers d'encre, Le jeu d'échecs et les lettres $\left(X I X^{e}-X X^{e}\right)$, sous la direction de J. Berschtold, Genève, Droz, 1998. Sur les poétiques ludiques, voir Désordres du jeu. Poétiques ludiques, études d'histoire et de littérature réunies par J. Berchtold, C. Lucken et S. Schoettkte, Genève, Droz, 1994.

${ }^{3}$ La machine littérature, Paris, Seuil, 1993.

${ }^{4}$ Sur ces textes, cf. F. Debor, «Lectures de sable ou les châteaux du sens. À propos du Château des destins croisés d'Italo Calvino », Désordres du jeu... ouvr. cit., p. 173-187.

Cahiers de Recherches Médiévales, 14, 2007 
d'Yvain en particulier, qui s'ouvre à la cour du roi Arthur, a Carduel en Gales/ après mangier, par mi ces sales, tandis que chevaliers et dames recontoient noveles ou parloient d'Amors ${ }^{5}$. Plus proche culturellement de Calvino, Boccace, au XIV siècle, offre un cadre semblable avec le Decameron : pour fuir la peste qui ravage Florence, une brigade composée de sept femmes et de trois hommes, nobles, beaux et richement vêtus, se réfugie dans un château situé "à l'écart de tous les chemins » et entouré «d'arbres aux abondantes frondaisons». Si la forêt de Calvino rappelle davantage la selva oscura de Dante ${ }^{6}$, les châteaux de Boccace et de Calvino ont en commun le raffinement et l'abondance qui en font des refuges de choix au milieu d'un univers chargé de menaces?. Mais ici Calvino fait subir une entorse au cadre. Tandis que chez Boccace, l'une des dames, Pampinée, invite la «joyeuse brigade » réunie dans un pré à passer les heures chaudes non giucando (...) ma novellando c'est-à-dire à délaisser les jeux, jeux de dames et jeux d'échecs, pour raconter chacun une histoire, chez Calvino, au moment de conter leur aventure, la parole fait défaut aux chevaliers. Cette absence de parole n'est pas à interpréter de façon psychologique, mais fonctionne comme une rupture mécanique, de l'ordre de la mutilation sociale: à la façon de l'inexistence du Chevalier inexistant, de l'arboricolisme du Baron perché, de la fracture en deux du Vicomte pourfendu, cette parole coupée est le point de départ de la fiction de Calvino\%. Il en déploie de façon logique toutes les possibilités, selon ce que Barthes appelle «la mécanique du charme $»^{10}$. Le fil coupé de la parole est en effet ici relayé par les fils narratifs que sont censées tisser entre elles les images d'un jeu de tarots. Il s'agira donc de passer les heures nocturnes giocando, novellando, à raconter en jouant ou en jouant à raconter.

\section{Jouer à raconter: le narrateur-joueur ou comment Calvino joue avec la figure du narrateur}

En effet, tandis que le narrateur 1 du récit-cadre cherche la parole qui lui manque pour raconter son histoire, celui qui paraît être le châtelain pose sur la table un jeu de tarots. L'un des convives initie la partie en tirant, dans le jeu, une carte dont la figure peinte lui ressemble. Avec la carte du Cavalier de Coupe se trouve dédoublée la figure de ce second narrateur. Au dire du premier narrateur, en effet: «Il nous parut qu'avec cette carte il voulait dire «je» et qu'il s'apprêtait à nous raconter son histoire $»^{11}$. À côté de cette première carte, fixant ainsi la règle, le

${ }^{5}$ Le Chevalier au lion (Yvain), publié par M. Roques, Paris, Champion, 1982, vv. 7-8 et 1213 .

${ }^{6}$ Dante, La Divina Comedia, chant I, l'Inferno.

${ }^{7}$ A. Frasson-Marin, Italo Calvino et l'imaginaire, Genève-Paris, Slatkine, 1986, p. 195-196.

${ }^{8}$ Giovanni Boccaccio, Decameron, Milano, Arnoldo Mondadori, 1996, p. 31. cf. Boccace, Le Décaméron, traduction de J. Bourciez, Paris, Garnier Frères, 1967, p. 25 : « Si vous suivez mon conseil, vous ne jouerez pas. (... ) Mais si chacun de nous nous contait une histoire, toute l'assistance en serait charmée...».

${ }^{9}$ G. Bertone, Il castello della scrittura, Torino, Piccola biblioteca Einaudi, 1994.

${ }^{10}$ R. Barthes, «La mécanique du charme », préface au Chevalier inexistant d'Italo Calvino, Paris, Seuil, 1984.

${ }^{11}$ P. 12. 
second narrateur appose successivement, une série de cartes à travers lesquelles les autres convives sont censés deviner son histoire. Ce dédoublement de la figure du narrateur est présent dans la littérature médiévale. Au début d'Yvain, l'auteurnarrateur prend la parole pour ensuite la déléguer à l'un des chevaliers d'Arthur, Calogrenant :

Il m'avint plus a de set anz

que je, seus come païsanz,

aloie querant aventures... ${ }^{12}$.

À la façon du second narrateur de Calvino, Calogrenant dispose les cartes de sa propre histoire: l'essart, le vilain, la fontaine etc..., tandis qu'après les interventions de $\mathrm{Keu}$, de la reine et d'Yvain, le récit raconte ensuite, à la troisième personne, l'aventure d'Yvain. Cet entrelacs des voix narratives est plus présent encore dans les récits en prose où l'auteur-narrateur délègue la parole au conte; la formule le conte dit que désignant à la fois la source écrite, d'où est censée partir la parole, et le récit même, entrain de se faire. Ce dédoublement est également celui de Blaise, le scribe, écrivant sous la dictée de Merlin ${ }^{13}$. Sur ce modèle, Calvino a déjà mis en scène, dans le Chevalier inexistant, un auteur-narrateur, sœur Théodora, qui s'avérait être l'un des personnages de l'histoire, Bradamante, projetant ainsi dans la fiction la figure de l'auteur ${ }^{14}$.

Dans Le Château, la figure d'auteur-narrateur est non seulement dédoublée, mais démultipliée. Une carte posée va faire réagir un autre convive qui va lui apposer une nouvelle série de cartes pour faire rebondir le récit dans une direction nouvelle : celle de sa propre histoire. Ainsi de suite, chaque chevalier va reconstituer à partir de celle des autres son histoire personnelle ; le narrateur 1 également, puis, à la fin de la première nouvelle, le châtelain-aubergiste et son épouse, et dans la Taverne, Calvino lui-même qui se met en scène comme un des personnages. Tandis qu'une immense histoire se compose, la figure de l'auteur-narrateur, démultipliée, s'est perdue dans le labyrinthe des histoires.

Tous à la fois, nous avançons les mains, vers les cartes, l'une des figures quand on la range auprès de plusieurs autres me fait revenir en mémoire l'histoire qui m'a amené ici, j'essaie de reconnaître ce qui m'est arrivé et de le montrer aux autres, qui pendant ce temps s'y recherchent aussi, dans ces cartes, et ils me montrent du doigt un tarot ou un autre, et rien ne va plus, et nous nous arrachons les cartes des mains, et nous les éparpillons sur la table ${ }^{15}$.

\footnotetext{
${ }^{12}$ Éd. cit., vv. 171-173.

${ }^{13}$ Cf Merlin, roman en prose du XIII ${ }^{\mathrm{e}}$ siècle, éd. Gaston Paris et Jacob Ulrich, SATF, Paris, 1886, mis en français moderne par E. Baumgartner, Paris, Stock, « Moyen Age », 1980, p. 6769.

14 «Dans l'acte d'écrire, le moi de l'auteur se dissout: ce qu'on appelle la «personnalité » de l'écrivain est intérieure à l'acte d'écrire, elle est un produit et un moyen de l'écriture». «Cybernétique et fantasmes. Ou de la littérature comme processus combinatoire», $L a$ machine littérature, ouvr. cit., p. 7-23.

${ }^{15}$ La Taverne, p. 61.
} 


\section{Raconter en jouant: le tarot de Milan ou comment Calvino joue de la matière médiévale comme réservoir d'images}

L'idée d'un Moyen Âge qui se donne à lire par les images n'est pas nouvelle. On pense aux vitraux, aux enluminures, mais également aux blasons, aux armoiries qui mettent en image l'identité individuelle et sociale pour l'offrir au déchiffrement. Ici, c'est un jeu de tarots peints par Bonifacio Bembo pour les ducs de Milan au milieu du $X^{\mathrm{e}}$ siècle, qui permet aux différents chevaliers de mettre en images leur histoire $^{16}$. Dans ce répertoire de figures, Calvino a lu tout d'abord le monde de l'Arioste :

La référence littéraire qui me vint spontanément à l'esprit fut le Roland furieux; même si les miniatures de Bonifacio Bembo précédaient de presque un siècle le poème de l'Arioste, elles pouvaient représenter très bien le monde visuel dans lequel l'imagination du poète s'était constituée. J'essayai aussitôt de composer, avec les tarots Visconti, des séquences inspirées du Roland furieux; il me fut facile de construire de cette façon la croix centrale des récits de mon «carré magique ». Il suffisait qu'autour prennent forme d'autres histoires qui se croisaient entre elles, et ainsi j'obtins une sorte de mots croisés faits de figures au lieu de lettres, où en plus chaque séquence peut se lire dans les deux sens ${ }^{17}$.

Le Moyen Âge, tel qu'il est parvenu à Calvino à travers le Roland furieux, constitue donc «la croix centrale» du «carré magique» du Château. Je rappellerai la trame de l'œuvre de l'Arioste, afin de montrer par quel procédé les différentes cartes du tarot Visconti la mettent en image. Dès le Roland amoureux de Boiardo, à la fin $\mathrm{du} \mathrm{XV}^{\mathrm{e}}$ siècle, Roland est épris, comme son cousin Renaut, d'Angélique, fille du roi du Cathay, envoyée par ce dernier pour rendre amoureux les paladins de Charlemagne et les détourner de la guerre contre les Maures. Avec le Roland furieux, au début du $\mathrm{XVI}^{\mathrm{e}}$ siècle ${ }^{18}$, l'Arioste prolonge ce récit en racontant comment Roland, par suite de son amour malheureux pour Angélique éprise de Médor, devient fou furieux au point de mettre en péril les armées chrétiennes privées de leur champion, et comment la raison perdue par Roland est retrouvée par Astolphe sur la Lune et remise dans le corps de son propriétaire qui réintègre sa place dans l'armée. Parallèle à celui-ci, un deuxième fil romanesque raconte les amours de Bradamante, la guerrière chrétienne, et de Roger, le champion du camp sarrazin.

Dans Le château, l'Histoire de Roland fou d'amour se dispose sur deux rangées de cartes horizontales au centre du jeu : Roland, le Roi d'Epée, est placé à hauteur du Dix d'Epée figurant l'armée de Charlemagne. Angélique apparaît sous les traits de la Reine d'Epée. La forêt à travers laquelle se cherchent les héros est représentée par l'entrelacs du Dix de Bâton. Le Chariot, conduit par une «dame en habits de magicienne...», figure la fuite d'Angélique à travers bois. C'est l'arcane de l'Amoureux qui révèle son étreinte avec Médor dont le portrait apparaît sur la carte du Valet de Bâton. La carte du Soleil, «un enfant qui court, le soleil sur la tête», est

\footnotetext{
${ }^{16}$ Plus encore aussi qu'un jeu d'échecs où les pions sont davantage réduits à des fonctions.

${ }^{17}$ Note, p. 136.

${ }^{18}$ Roland furieux, traduction de C. Hippeau, présenté et raconté par I. Calvino. Traduction de Nino Frank, Paris, Flammarion, 1982.
} 
interprétée comme la raison envolée de Roland. «La Force, énergumène terrassant un lion avec une massue », met en image la furie du héros, tandis que le Cinq de Bâton dessine le tronc des chênes qu'il a déracinés. Loin du monde guerrier, Durandal abandonnée attachée à un arbre apparaît dans le Sept d'Epée, tandis que la raison perdue de Roland est, comme le rappelle le Trois de Coupe, sur la Lune, «à l'intérieur d'une ampoule gardée dans la Vallée des Raisons Perdues ». La carte du Fou figure alors Roland avec tous les attributs du fou au Moyen Âge: "sa massue sur l'épaule à la façon d'une lance, maigre comme un squelette, déguenillé, ses braies perdues, avec la tête pleine de plumes....». La carte de la Justice, enfin, représente les chevaliers de Charlemagne sur les traces de leur champion, afin de « ramener son épée au service de la Justice et de la Raison», et figure par cela même, également, la Raison. La toute dernière carte, le Pendu, représente Roland attaché la tête en bas et disant : «laissez-moi comme ça. J'ai fait le tour et j'ai compris. Le monde se lit à l'envers. Voilà. » Croisant l'histoire de Roland, celle d'Astolphe sur la lune se dispose sur les deux rangées verticales centrales du jeu. Les séquences du roman de l'Arioste servent ainsi de charpente à la construction des autres histoires.

La littérature médiévale fonctionne alors comme un répertoire d'images susceptible de construire toutes les autres histoires. À rebours, on pourrait s'amuser à lire ainsi toute la littérature médiévale. Les motifs qui jalonnent l'histoire de Calogrenant - la forêt, le vilain à la massue, la fontaine, le combat - pourraient être figurés comme suit: la forêt, par la carte du Neuf de bâton « avec son enchevêtrement de branches »; le vilain par La Force, "un énergumène armé...une masse brandie en l'air»; la fontaine par L'As de coupe "sur lequel était dessinée une fontaine dont l'eau courait parmi les mousses fleuries et les battements d'ailes»; tandis que le chevalier de la fontaine pourrait être Le Cavalier d'épée. Il ne reste plus à Yvain qu'à rebattre les cartes de Calogrenant pour élaborer sa propre histoire : Le Neuf de bâton, La Force, L'As de coupe, Le Cavalier d'épée, puis, pour figurer Laudine, L'impératrice, "une épouse de haut et opulent lignage «, et enfin le Fou, carte toujours "exceptionnellement adéquate au propos", comme dit Calvino, pour représenter la folie d'Yvain. De cette façon pourrait-on résumer, sans doute, toute la littérature médiévale.

\section{La littérature médiévale comme jeu de cartes?}

Calvino ne retient-il alors du Moyen Âge qu'un simple jeu de cartes aux images diversement battues et rebattues? Un bric à brac de motifs comme autant de vignettes sorties, éparses d'un album, ou d'armoiries, d'un armorial? La Taverne se clôt sur ces paroles de Macbeth :

Je commence à être las du Soleil et souhaite que se casse la syntaxe du monde : que se mêlent les cartes, les feuilles de l'in-folio, les fragments de ce miroir du désastre ${ }^{19}$.

Ces images, décrochées d'une trame narrative précise, risquent de perdre leur sens. En panne de sens, en quête d'histoires, de drame, elles sont tels les

${ }^{19}$ La Taverne, p. 131. 
personnages de Pirandello " comme déjà détachés de leur support narratif, personnages d'un roman sortis par miracle des pages du livre qui les contenait... $»^{20}$ :

Et ils attendaient là, chacun avec son tourment secret et tous unis par leur naissance et par l'enchevêtrement de leurs mutuelles vicissitudes, que je les fisse entrer dans le monde de l'art, en composant de leurs personnes, de leurs passions et de leurs aventures un roman, un drame, tout au moins, une nouvelle ${ }^{21}$.

Si ces derniers étaient en quête d'auteurs, les images sont, elles, en quête de lecteurs. Seul les lecteurs peuvent lire dans leur désordre les lignes des histoires et échafauder avec coupes, deniers, bâtons et épées, chariot, force, fou..., les pans d'un château de cartes. Conformément aux perspectives critiques de son temps, la littérature médiévale n'offre pas seulement un album d'images à Calvino, mais également un modèle d'art et de lecture du récit.

\section{La littérature médiévale comme château de cartes}

\section{Le récit comme le château de cartes}

À travers le narrateur I, Calvino explicite ainsi sa démarche :

En fait, la tâche de déchiffrer les histoires une à une m'a fait jusqu'à présent négliger la particularité la plus saillante de notre mode de narration, à savoir que chaque récit court à la rencontre d'un autre, et tandis qu'un des convives progresse sur sa lancée, un autre parti de l'autre bout avance en sens opposé, puisque les histoires racontées de gauche à droite ou de bas en haut peuvent aussi bien être lues de droite à gauche ou de haut en bas, et vice versa, si on tient compte du fait que les mêmes cartes, en se présentant dans un ordre différent, changent de sens, et que le même tarot sert dans le même temps à des narrateurs qui partent des quatre points cardinaux ${ }^{22}$.

C'est à l'occasion d'un séminaire international sur les stratégies du récit, à Urbino en 1969, dans lequel Paolo Fabri faisait une communication sur «le récit de la cartomancie et le langage des emblèmes ", que Calvino eut l'idée d'employer les tarots « comme machine narrative combinatoire ». Cette aptitude des tarots à illustrer la combinatoire du récit se double du fait que leurs images mettent en scène le Moyen Âge et la matière médiévale. De surcroit, le Moyen Âge lui-même a pensé l'art de la combinatoire, avec l'ars combinatoria, théorisé dans L'Ars Magna de Raimond Lulle, auquel Calvino fait allusion dans «Cybernétique et fantasmes $»^{23}$. Lulle pensait qu'il existait dans chaque domaine de la science quelques principes fondamentaux et proposait de combiner toutes les sciences humaines à partir de ces données élémentaires. À cet effet, il ordonnait les neuf principes fondamentaux sur

\footnotetext{
${ }^{20}$ Six personnages en quête d'auteurs, Préface. Cf. Pirandello, Théâtre complet, t. 1, Paris, Gallimard, Pléiade, p. 998.

${ }^{21}$ Ibid., p. 996.

${ }^{22}$ Le Château, p. 47.

${ }^{23}$ La Machine littérature, «Cybernétique... », art. cit., p. 11.
} 
le pourtour d'un cercle, ainsi que sur un deuxième cercle plus petit, ou d'autres encore ; les différents cercles pouvant se mouvoir les uns par rapport aux autres. La simple rotation de ces «cercles conceptuels» permettait de créer de nouvelles combinaisons qu'il suffisait de compléter en fonction du domaine d'application. Les «vérités» conceptuelles naissaient ainsi mécaniquement. Le récit médiéval peut également relever de ce principe combinatoire. On peut lui appliquer ce que Calvino dit de la narration orale primitive :

(Elle) se modèle sur des structures fixes, comme sur des éléments qu'on pourrait dire préfabriqués, qui permettent cependant un nombre immense de combinaisons... des permutations et des transformations illimitées ${ }^{24}$.

Calvino illustre cela lorsque, croisant l'histoire de Faust et celle de Perceval, il met en image quelques motifs du Conte du Graal. Perceval est le Cavalier d'Epée, enfant de la gaste forest dont les terres désolées sont représentées par la Lune, et qui devra faire des détours, "comme l'indique le signe serpentin du Deux de Deniers", pour atteindre le château du Roi Pêcheur, ici La Maison-Dieu, où séjourne Amfortas, représenté par le Roi de Deniers «se touchant une plaie qui ne se referme pas »; quant au graal qui laisse muet, c'est l'As de Coupe qui le figure. Or le Conte $d u$ graal a engendré un autre type de textes qui fonctionnent à partir du procédé de la bifurcation. Ce sont les «continuations" dont la vocation est de donner une suite au Conte du graal. Si la Première continuation se greffe directement sur les derniers vers de Chrétien, la seconde se greffe à l'endroit même où le héros se sépare de l'oncle-ermite, - à la carte Ermite donc-, dans l'intention de retrouver le graal. Ce récit, construit autour de la quête du graal, repousse sans cesse son achèvement par les procédés récurrents du détour et du brouillage des pistes narratives ${ }^{25}$. Perceval, à plusieurs reprises, est détourné de la «droite voie» pour se retrouver, par exemple, dans le château de l'Echiquier Magique. De même, la quête féerique de la tête du blanc cerf vient concurrencer la quête chrétienne du saint graal; comme si, au dernier moment, le narrateur disposait sur la mosaïque du récit une carte inattendue qui le ferait bifurquer.

En choisissant, à travers les cartes d'un tarot du $\mathrm{XV}^{\mathrm{e}}$ siècle, la matière médiévale pour illustrer une définition de la littérature comme «la permutation d'un ensemble fini d'éléments et de fonctions ${ }^{26}$, Calvino s'inscrit au cœur de la pensée structurale, telle qu'elle ressort des analyses de Propp - selon lesquelles toutes les fables sont les variantes d'une même fable et peuvent se décomposer en un certain nombre de fonctions narratives- et telle qu'elle inspire l'investigation littéraire pratiquée par l'Ouvroir de littérature potentielle, avec, entre autres réalisations, les Cent mille milliards de poèmes de Queneau. Cet ouvrage, dit Calvino, « est moins un livre qu'un modèle rudimentaire de machine à construire des sonnets différant tous les uns des autres $»^{27}$. Sur ce modèle, le château de cartes peut tout entier être pensé comme "une machine à construire des nouvelles»; une machine capable de se

\footnotetext{
${ }^{24}$ Ibid., p. 8.

${ }^{25}$ M. Séguy, Les Romans du Graal ou le signe imaginé, Paris, Champion, 2001, p. 303-315.

${ }^{26}$ La Machine littérature, « Cybernétique... », art. cit., p. 17.

${ }^{27}$ Ibid., p. 13.
} 
passer d'auteur ou plutôt, projetant, dispersant au fil des cartes la figure de l'auteur comme «simple stratégie textuelle» pour reprendre les termes d'Umberto Eco $^{28}$, ou, pour reprendre ceux du premier narrateur:

Il ne reste plus de moi que l'obstination maniaque de compléter, de fermer, de faire revenir les récits ${ }^{29}$.

Utiliser la matière médiévale comme matériau de cette réflexion sur la production du texte, c'est alors retrouver un état de texte où l'auteur n'est pas tout à fait encore, pour écrire une œuvre où l'auteur n'est plus. Car,

«Le processus de la composition littéraire une fois démonté et remonté, le moment décisif de la littérature deviendra la lecture... Ce qui disparaîtra ...ce sera la figure de l'auteur $»^{30}$.

\section{Au lecteur de jouer}

Dans le Château des destins croisés, l'effacement de l'auteur va de pair avec sa dispersion, sa démultiplication à travers les figures des différents chevaliers. Chacun est tour à tour narrateur et lecteur; à peine un chevalier-narrateur tente-t-il de reconstituer son histoire - ou la donne-t-il à reconstituer- qu'un autre chevalierlecteur, discernant dans l'histoire de l'autre les linéaments de sa propre histoire, s'en empare pour raconter la sienne. Et ainsi de suite. La narration est comme avalée par le déploiement de la lecture et vice versa.

À chaque nouvelle carte que le jeune homme essaie de disposer auprès des autres, dix mains s'allongent pour la lui prendre et la placer dans une autre histoire que chacun veut exposer, et à un certain point les cartes lui échappent des mains de tous côtés, il doit les retenir avec ses mains, ses avant-bras, ses coudes, et de la sorte il les cache même à qui voudrait comprendre l'histoire qu'il est, lui, en train de raconter ${ }^{31}$.

Narration et lecture se disputent la partie, s'engendrent l'une l'autre, se déroulent et s'enroulent à la façon des deux faces sans cesse réversibles d'un ruban de Moebius. Mais s'il y a autant de lecteurs que de narrateurs, c'est qu'il y aussi autant de lectures que de lecteurs; et les rubans des histoires de se dédoubler, de s'emmêler. La multiplication des lecteurs est l'image de la pluralité des lectures. Un lecteur pluriel, c'est ainsi qu'il faut entendre ici le "Lecteur Modèle» dont parle Umberto $\mathrm{Eco}^{32}$. Seul ce lecteur pluriel saura lire dans les cartes et donner «leur(s) vraie(s) vie(s)» aux histoires. La carte du Monde sera tour à tour «cité céleste», «cité fortifiée assiégée», «Paris convoité par les Maures» ou «Troie assiégée», ou

${ }^{28}$ U. Eco, Lector in fabula, Paris, Grasset, 1985, p. 79.

${ }^{29}$ Le Château, p. 53.

${ }^{30}$ Art. cit., p. 15.

${ }^{31}$ La Taverne, p. 73.

${ }^{32}$ C'est-à-dire un lecteur « capable de coopérer à l'actualisation textuelle de la façon dont lui l'auteur le pensait, et capable d'agir interprétativement comme lui a agi générativement », U. Eco, ouvr. cit., p.71. 
encore « ville souterraine». Pour le lecteur cartomancien, la lecture est divination des possibles, à la fois maîtrise du devenir des histoires et surprise du surgissement du sens. En ce sens, plus que les échecs, le jeu de tarot permet d'associer l'écriture/ lecture à la divination; association fréquente dans la littérature médiévale dans laquelle les actes de dire et de prédire le récit se confondent, et où le lecteur professionnel peut à son gré conter, rajouter, supprimer, au moyen même, parfois, d'une suite d'images comme support mémoriel d'un récit à réinventer. Dans cet exercice divinatoire auteur et lecteur rivalisent, chacun voulant imposer à l'autre sa vision propre de l'histoire à écrire ou à lire.

\section{Le récit comme château-labyrinthe}

Plus les histoires deviennent embrouillées et tirées par les cheveux plus les cartes ainsi éparpillées trouvent leur place dans une mosaïque bien rangée. Est-ce seulement le fait du hasard, ce dessin, ou bien l'un d'entre nous n'en construit-il pas avec patience l'assemblage ${ }^{33}$ ?

Cette mosaïque si bien rangée, où se perd toutefois le chemin des histoires, offre l'image d'un labyrinthe dont seul le lecteur patient pourrait construire et déconstruire l'assemblage. Comme le dit Calvino :

Le labyrinthe est fait pour qu'on s'y perde et qu'on y erre. Mais il est aussi un défi au visiteur, pour que celui-ci en reconstitue le plan et en détruise le pouvoir. S'il réussit, il aura détruit le labyrinthe ; le labyrinthe n'existe pas pour qui l'a traversé.

Le labyrinthe n'existe pas pour le lecteur cartomancien qui sait faire courir le fil du sens dans le dédale du château de cartes.

Là encore, le récit médiéval peut aussi offrir la structure du labyrinthe. C'est même la métaphore topique qui permet de rendre compte des grands romans en prose du XIII ${ }^{\mathrm{e}}$ siècle. Les multiples voies de la forêt y sont comme autant de voies ouvertes au récit où le lecteur court le risque de se perdre. La technique de l'entrelacement ${ }^{34}$ permet d'entrecroiser les différents fils narratifs. Mireille Séguy a montré comment le Perlesvaus, par delà la rigueur de sa composition et de sa division en onze branches, relève moins de la structure de l'arbre que de celle d'une série d'aventures inextricablement enchevêtrées les unes aux autres, tantôt suspendues, tantôt indéfiniment prolongées dans des ramifications secondaires. «Un personnage secondaire peut resurgir dans une autre branche, dans un rôle d'adjuvant ou d'opposant, selon la nature des rapports qu'il a précédemment noués avec le héros $»^{35}$. On retrouve l'idée des cartes qui apparaissent avec des sens différents. De même, le récit peut passer d'un personnage principal à un autre par le biais d'un espace-relais - cour d'Arthur, ermitage- ou d'un personnage-relais, ou bien par la rencontre directe d'un nouveau personnage dont on va suivre les aventures en

\footnotetext{
${ }^{33}$ La Taverne, p. 98.

${ }^{34}$ Terme employé pour la première fois à propos du Lancelot en prose. Cf. F. Lot, Étude sur le Lancelot en prose, p. 17.

${ }^{35}$ M. Séguy, ouvr. cit., p. 344.
} 
abandonnant celles du premier ${ }^{36}$. La ramification des aventures secondaires, la multiplication des personnages génèrent donc un texte «proliférant et mouvant, ou, pour le dire autrement, labyrinthique $»^{37}$.

Chez Calvino, si le récit est bien un labyrinthe, le livre tout entier en prend également la forme, comme c'est le cas chez Borges, dans le Jardin aux sentiers qui bifurquent. Dans cette nouvelle, le narrateur - dont l'arrière grand-père a passé sa vie à écrire un roman et à composer un labyrinthe- poursuivi, se réfugie dans la demeure du professeur Stephen Albert. Ce dernier va lui montrer le labyrinthe de son aïeul. Le narrateur se trouve tour à tour face à un jardin, puis à un secrétaire en laque, « un labyrinthe en ivoire ", puis à un livre, "un labyrinthe de symboles », jusqu'à ce qu'il comprenne que "le livre et le labyrinthe étaient un seul objet», lieu de tous les dénouements possibles, de toutes les bifurcations ${ }^{38}$. Sous la plume de Borges, le labyrinthe prend aussi l'image de la bibliothèque de Babel aux galeries infinies ${ }^{39}$. Dans cette dernière métaphore, l'association est explicite : le labyrinthe est une des figures de l'univers. Le château de Calvino, lieu de toutes les images, de toutes les figures et de toutes leurs combinaisons possibles, n'est pas loin de la bibliothèque de Borgès qui «comporte toutes les structures verbales, toutes les variantes que permettent les vingt-cinq symboles orthographiques...»; à l'image du labyrinthe, il est «un fac similé du monde».

Mais, Calvino dit: «le tour de passe-passe qui consiste à aligner des tarots pour en tirer des histoires, je pourrais aussi le réussir avec des peintures de musées »; pourquoi alors l'imagerie médiévale pour signifier la combinatoire du récit? Pourquoi pas, comme il en avait eu aussi l'idée, la bande dessinée, «avec gangsters, femmes terrorisées, astronefs, vamps, guerre aérienne, savants fou $»^{40}$ ? C'est que dans cette expérience, le Moyen Âge dans son ensemble est bien « la carte à jouer » par excellence, non seulement pour illustrer les contours mouvants de l'œuvre ouverte, mais aussi pour tendre au lecteur la carte tantôt obscure, tantôt claire, de ses rêves.

\section{Le Moyen Âge comme «le dessous des cartes»}

\section{Le Moyen Âge comme carte-sens}

Jouer la carte du Moyen Âge, c'est aller rechercher, sous les cartes, la carte du sens. Dans le jeu de tarot, c'est le Deux de deniers qui la symbolise dans «Moi aussi je veux raconter la mienne ». L'auteur-narrateur l'interprète ainsi :

Le Deux de deniers est pour moi (...) la lettre qu'il ne faut pas prendre à la lettre, la lettre qui transvalue les valeurs qui sans lettre ne valent rien, la lettre toujours prête à grandir de son propre mouvement et s'orner des fleurs du sublime, tu la vois là historiée et fleurie en sa face signifiante, élément premier des Belles Lettres, et qui

${ }^{36}$ Ibid., p. 345-346.

${ }^{37}$ Ibid., p. 357.

${ }^{38}$ Borges, CEuvres complètes, Fictions, Pléiade, Gallimard, 1999, vol. I, p. 506.

${ }^{39}$ Borges, ibid., p. 491.

${ }^{40}$ Note, p. 144. 
enveloppe dans ses spirales la circulation du signifié, $\mathrm{S}$ qui serpente pour signifier qu'elle est là toute prête à signifier les signifiés, signe signifiant qui revêt la forme d'un S pour que ses signifiés prennent eux-mêmes la forme de petits $s$.

Tout droit sortie d'un manuscrit médiéval, la carte du sens est la carte séduisante qui promet la transfiguration, la sublimation du réel. Le Moyen Âge comme carte du sens répondrait ainsi à la quête des origines. Il constituerait la carte où s'inscrit l'origine; l'origine d'un avènement du sens, l'origine de son interprétation et l'origine de toutes les histoires que ce sens traverse.

Âge de l'avènement du sens, le Moyen Âge représente l'âge où les choses, les figures adviennent à elles-mêmes, trouvent leur sens. Dans le Chevalier inexistant, la sœur Théodora décrit ainsi l'époque à laquelle se déroule son histoire :

L'ordre de l'univers, en cet Âge ancien où se déroule mon histoire, était encore plein de hasards. Plus d'une fois, il arrivait qu'on se trouvât mis en présence de vocables, de notions, d'institutions et de formes à quoi ne correspondait rien de réel; en revanche, le monde regorgeait de choses, d'énergies et d'êtres que rien, pas même un nom, ne différenciait du reste (...). Et quelquefois, par l'effet du hasard ou d'une intuition, cette masse venait se loger dans un nom, un titre nobiliaire (...) en une case de l'organigramme militaire... et puis, surtout, dans une armure inoccupée $e^{41}$.

Si l'on identifie le Moyen Âge à cet Âge ancien, ce dernier figure comme une image du chaos initial, un état antérieur du monde où les mots et les choses ne se seraient pas encore trouvés; un état de choses toutes dans la ressemblance et la continuité, avant l'apparition du langage comme facteur de distinction et de sens. Plus exactement, le Moyen Âge figure le moment où les choses commencent à trouver leur nom, les figures, leur sens ; les corps, leur armure. Le Moyen Âge serait l'âge de ce passage, de cet avènement au sens ; un âge non pas tant d'enfance, mais d'adolescence du monde et du sens. Faire le détour par cette époque pour illustrer la combinatoire du récit, c'est aller chercher, en somme, le dessous des cartes, se livrer à une archéologie du sens, rechercher l'âge où le sens vient aux images.

Or c'est dans un travail du sens, dans le déchiffrement des signes, dans la tentative de nommer, d'interpréter, que le sens vient aux images. Le Moyen Âge figure par excellence l'âge où l'on apprend à lire le monde. La carte du Roi de Deniers est interprétée ainsi :

il était entré en possession d'un héritage considérable et s'était aussitôt mis en voyage. Nous déduisîmes cette dernière proposition du mouvement de bras qu'il eut pour jeter la carte du Neuf de Bâton, laquelle - avec son enchevêtrement de branches posées sur une rare végétation de feuilles et de petites fleurs des champs- nous rappelait le bois que nous venions de traverser ${ }^{42}$.

\footnotetext{
${ }^{41}$ I. Calvino, Le Chevalier inexistant, ouvr. cit., chap. IV, p. 41.

${ }^{42}$ Le Château, p. 14.
} 
De l'enchevêtrement des branches et des signes se déduit non pas l'ébauche d'un sens, mais son foisonnement. De fait, le Moyen Âge, âge de l'interprétation, est par définition l'âge des interprétations multiples. Chaque carte fait ainsi l'objet, dans chacune des histoires, d'une interprétation différente. Le sens est bien ce qui circule, "serpente» entre les signifiés. Mais Calvino joue également sur les différents niveaux d'interprétation, littéral et allégorique, tels que les pratiqua, à partir de la lecture de la Bible, l'exégèse médiévale ${ }^{43}$. L'interprétation de la carte du Soleil se discute :

Le peintre avait représenté l'astre du jour entre les mains d'un enfant qui court, ou même qui vole, au-dessus d'un paysage vaste et varié. L'interprétation de ce passage du récit n'était pas aisée; ce pouvait vouloir simplement dire «c'était par une belle journée ensoleillée» (...). Mais peut-être convenait-il de s'arrêter, plutôt qu'à la signification allégorique, au sens littéral : un enfant à demi-nu avait été vu courant dans le voisinage du château où l'on célébrait les noces, et c'était pour suivre ce gamin que le marié avait déserté le repas ${ }^{44}$.

C'est donc par couches successives que le sens vient aux images ; si ce n'est que l'avènement du sens mis en scène par Calvino donne à la fois le sens passé de l'histoire et les sens à venir du récit; la lecture, là encore, est inséparable de la divination. La Queste del saint graal en offre un exemple particulièrement éclairant : chaque aventure des chevaliers ne prend son sens que lorsqu'elle est glosée par la parole d'un ermite qui l'interprète en y mettant à jour l'avenir de la chevalerie et l'avenir du récit; comme si le chevalier jetait les cartes de son aventure qui ne devait prendre sens que par la lecture d'un saint homme. Le Moyen Âge apparaît donc comme l'âge où, en couches successives, le sens se dépose, se cristallise sur les images; cristal du sens où se lit l'avenir du récit, ou plus simplement, la naissance des histoires et de toutes les autres histoires.

À l'origine de toutes les histoires, dit Calvino dans la préface de la trilogie ${ }^{45}$, une image. Ici, à l'origine du château des histoires, un jeu de cartes ; un jeu de tarots du quattrocento pour mettre en image l'origine des histoires, comment naissent les histoires ou comment, cette fois, l'histoire vient aux images. Les cartes à faire jouer permettent de mettre en scène le souffle du conteur, les terreurs qui l'agitent, de la sortie de la forêt obscure jusque sur la table illuminée, ornée de couverts et de figures, qui les donne à lire. Une fois sur la table, les histoires deviennent si signifiantes, si exemplaires, qu'elles fonctionnent comme les germes de toutes les histoires possibles. C'est ainsi que l'histoire de Roland, charpente du "carré magique » de Calvino, donne naissance à toutes les autres histoires ; à celle de Faust, mais aussi à celle du voleur de tombes, de l'ingrat puni, de la femme damnée, de

${ }^{43}$ Cette dernière distingue plus particulièrement quatre niveaux d'interprétation: une interprétation littérale, une interprétation allégorique, une interprétation morale et enfin une interprétation anagogique. Voir, H. de Lubac, Les quatre sens de l'Écriture et M. Fiedrowicz, Principes de l'interprétation de l'Écriture dans l'Église ancienne, Peter Lang, 1998.

${ }^{44}$ Ibid., p. 17.

${ }^{45}$ I nostri antenati, Torino, Enaudi, 1960, p. 5. 
l'aubergiste, du guerrier mélancolique, ou du quidam, qui tous se disputent les mêmes cartes de la justice, des épées et des ermites, de l'amoureux et de la mort, du diable et de la lune. C'est que d'histoire en histoire s'écrit toujours la même histoire. Calvino fait ressortir un fonctionnement essentiel de l'écriture médiévale. Sans ambition d'originalité, elle se constitue dans l'adaptation et la réécriture d'histoires déjà écrites. De même, ici, l'histoire individuelle se perd dans la foule des histoires. "La forêt, le château, les tarots m'ont conduit à ceci, dit le narrateur : à perdre mon histoire, à la confondre dans la poussière des histoires, à m'en libérer $»^{46}$. Si exemplaires qu'elles contiennent en elles-mêmes toutes les autres histoires, les histoires des tarots opèrent chez leur lecteur une véritable catharsis.

L'écriture avertit de tout ça comme l'oracle, et comme la tragédie elle en purifie. En somme, il n'y a pas là de quoi faire un drame. L'écriture en somme possède un soussol qui appartient à l'espèce, ou du moins à la civilisation, du moins dans les limites de certaines catégories de revenus ${ }^{47}$.

Si l'écriture parle du «sous-sol» de l'espèce ou de la civilisation, les tarots enluminés font parler ce dessous des cartes de l'âme humaine et du récit que le Moyen Âge met en image. Ainsi, c'est le Moyen Âge dans son ensemble, comme musée des horreurs ou immense boutique de farces et attrapes, qui constitue le décor de la carte du Diable. Ce devrait être, dit l'auteur-narrateur,

la carte que dans mon métier on rencontre le plus souvent: la matière de l'écriture n'est-elle pas toute dans la remontée au jour de griffes velues, crocs canins, cornes caprines, violences interdites qui pataugent dans le noir ${ }^{48}$ ?

Le Moyen Âge, donc, comme image d'un inconscient du texte, image de ce que le texte charrie à son insu et qui « remonte au jour», lorsque justement l'on joue à combiner les images. Le Moyen Âge en même temps comme lieu chargé des fantasmes individuels et collectifs, qui parle à travers les cartes :

Tout cela est comme un rêve que la parole porte en soi et qui, passant par celui qui écrit, se libère en le libérant. Dans l'écriture, ce qui parle c'est le refoulé. Mais alors Le Pape à la barbe chenue ce pourrait être le grand pasteur d'âmes, l'interprète des songes, Sigismond de Vienne, et pour en avoir confirmation il n'y a qu'à vérifier si quelque part dans le tableau des tarots on peut lire l'histoire qui, à ce qu'enseigne sa doctrine, se cache dans la trame de tous les récits sans exception ${ }^{49}$.

$\mathrm{Si}$ «la ligne de force de la littérature moderne, comme le dit Calvino, tient dans sa volonté de donner la parole à tout ce qui, dans l'inconscient social et individuel, est resté non exprimé ${ }^{50}$, le Moyen Âge est bien la carte à jouer : carte-

\footnotetext{
${ }^{46}$ Le Château, p. 53.

${ }^{47}$ La Taverne, p. 114.

${ }^{48}$ Ibid., p. 111.

${ }^{49}$ Ibid., p. 112.

${ }^{50}$ La Machine littérature, art. cit., p. 18.
} 
sens, carte pleine ou plutôt obscurcie de nos angoisses et de nos rêves, carte à remplir.

\section{Le Moyen Âge comme carte vide}

Le Moyen Âge permettrait de mettre en scène non seulement le moment où les choses trouvent leur sens et les images leurs histoires, mais aussi, dans le même mouvement, le moment où celui qui a appris à lire, à déchiffrer, advient à lui-même. Il fonctionnerait comme la carte vierge de toute conscience de soi, où peu à peu se reconnaissent et se dessinent des visages, où s'inscrit un destin. Dans la préface à la trilogie ${ }^{51}$, Calvino affirmait son désir que ces trois histoires «puissent être regardées comme un arbre généalogique des ancêtres de l'homme contemporain». Agilulfe, le Chevalier inexistant, le Vicomte pourfendu et plus tard dans le temps, le Baron perché, en quête d'eux-mêmes, seraient ainsi nos pères, nos pères dans l'adolescence de leur être. On pourrait interpréter de la même façon les figures de tarot comme nos portraits de famille. La fébrilité avec laquelle les chevaliers recomposent, grâce aux cartes, leur histoire, nous les montre en quête d'eux-mêmes, de leur destin, de leur drame, dirait Pirandello. Les personnages se constituent devant nous. Il s'agit de pouvoir dire «je» et de remplir à coup de cartes ce programme. Le Moyen Âge figure alors comme un âge de l'avènement au «je». Le personnage de la littérature médiévale qui illustre par excellence la quête de soi, c'est bien Perceval, qui tente d'advenir à lui-même par le déchiffrement des images et le travail de la mémoire. Ici aussi c'est bien par le seul jeu de la mémoire que les personnages tentent de récupérer leur histoire ; une histoire qui immédiatement leur échappe, n'est plus que le reflet d'une autre histoire. «Je»n'est peut-être que le reflet d'un autre.

Si au milieu du Château, «les tarots disposés sur la table formaient un tableau complètement fermé, avec au centre une fenêtre restée vide ${ }^{52}$ », à la fin de la nouvelle, la grille est couverte par les tarots et les récits». En revanche, au centre de la mosaïque que constitue la Taverne, demeure un rectangle vide. Or, le Moyen Âge fournit lui-même des images de centre à la fois plein et vide. De moment où les formes trouvent leur sens, les images leur interprétation, le Moyen Âge finit par devenir l'âge où pullulent les formes-sens, les images recouvertes de sens. Le travail de Calvino est alors de recourir à ces formes, à ces images pour mieux les vider de leur contenu sémantique. L'espace du château, la cour d'Arthur, la Table Ronde, l'armure même de ses chevaliers, le graal enfin, sont dans la littérature médiévale des formes sens, dont Calvino dévide le sens, pour leur restituer le vide fécond qui les anime ${ }^{53}$. Le château, envisagé d'abord comme opposé à la forêt, peut fonctionner aussi comme centre de cette forêt. Si les multiples châteaux des romans médiévaux démultiplient le centre de la forêt, font que son centre est nulle part, il y a bien un château qui figure le centre convoité et impossible à atteindre, c'est le château du Roi Pêcheur, le château du graal. Le château lumineux des destins croisés peut

\footnotetext{
${ }^{51}$ Ouvr. cit., p. 5.

${ }^{52}$ Le Château, p. 35.

${ }^{53} \mathrm{Cf}$. D. Demartini : «Le Chevalier inexistant: l'écriture médiévale rouage de la 'machine littérature' d'Italo Calvino ", Images du Moyen Âge, sous la direction d'I. Durand-Le Guern, PUR, 2006, p. 123-136.
} 
figurer ce château où la parole fait défaut pour dire, et où il s'agit justement de trouver, en se livrant au déchiffrement d'un cortège d'images - lance qui saigne, graal que l'on sert, tailloir- une autre façon de dire, afin que

le dépôt des siècles coagulé au fond des vases des fouilles se libère, les ères écrasées entre les couches telluriques recommencent à se dérouler, le futur récupère le passé, le pollen des saisons d'abondance depuis des millénaires enterré dans les tourbières s'envole de nouveau, s'élève par-dessus la poussière des années de siccité... ${ }^{44}$

Au centre de cet espace du déchiffrement qu'est le château, l'autre formesens retravaillée par Calvino n'est autre que le graal. Tandis que dans la littérature médiévale, le graal contient le sens, l'origine de toute chose, il est ici totalement vidé de son contenu sémantique. Dans le Chevalier inexistant, il se confond avec le tout. Ses chevaliers sont décrits comme une bande de fanatiques «cheminant à pas lents, la bouche ouverte, les yeux écarquillés «. À travers les chevaliers du graal ayant abandonné «tout vestige de vouloir humain», Calvino a voulu montrer "l'existence en tant que dissolution dans le tout», une autre version d'Agilulfe et de Gourdoulou. Le graal est figure du tout comme vide. Il décline l'image matricielle de la fiction de Calvino: l'armure d'Agilulfe illustrant le vide de l'homme contemporain. Ici, dans «Deux Histoires où l'on se cherche», Faust et Parcifal, à vouloir donner un sens à leur histoire, oscillent entre deux interprétations : tout et rien $^{55}$. La conclusion de Faust, dont la quête était l'immortalité, est la suivante :

Le monde n'existe pas, conclut Faust (...), il n'existe pas un tout donné en une fois : il y a un nombre fini d'éléments dont les combinaisons se multiplient par milliards de milliards, et parmi lesquelles quelques unes seulement trouvent une forme et un sens, qui s'imposent au milieu de la poussière insensée et sans forme; comme les soixante huit cartes du jeu de tarots dans les rapprochements desquelles apparaissent des séquences de récit qui aussitôt se défont.

De même, la conclusion de Perceval, dont la quête était le graal :

Le noyau du monde est vide, le principe de ce qui se meut dans l'univers c'est l'espace du rien, ce qui existe se construit autour d'une absence, au fond du graal il y a le tao.

Et il montre le rectangle vide, au centre, qu'entourent les tarots.

Le graal, comme le tao, qui d'ailleurs rime avec tarot, est donc la coupe réversible du tout et du rien, coupe à la fois pleine et vide. Au cœur du monde donc, le vide, un vide qui fait l'impasse sur la révélation transcendante. Sur la carte du sens, le « $\mathrm{S} »$ s'est inversé. Les courbes qu'il dessine sont celles du serpent; ses promesses ne peuvent tenir. Est-ce à dire que toute quête se réduit au néant et que la carte du Moyen Âge n'est jouée que pour mettre ce néant en images ? La lune d'Astolphe conforte et complète cette conclusion. «Sur les blanches étendues de la

\footnotetext{
${ }^{54}$ La Taverne, p. 106.

${ }^{55}$ Ibid., p. 106-107.
} 
Lune» où il est parti rechercher la raison perdue de Roland, Astolphe rencontre le poète,

appliqué à interpoler dans sa machine à tisser les rimes des strophes, les filets des intrigues, les raisons et les déraisons. Si celui-là habite au beau milieu de la Lune ou en est habité, comme par son noyau le plus profond -, il nous dira s'il est vrai qu'elle contient le dictionnaire universel des rimes, des mots et des choses, si elle est le monde plein de sens, l'opposé d'une Terre insensée.

Non, la Lune est un désert.

Telle était la réponse du poète, à en juger d'après la toute dernière carte tombée sur la table : la chauve circonférence de l'As de Deniers.

«De cette sphère aride part tout discours et tout poème; et chaque voyage à travers forêts batailles trésors banquets alcôves nous ramène ici; au centre vide de tout horizon. ${ }^{56}{ }^{\prime}$

Il s'agit bien de répondre à la question que pose Calvino dans «Cybernétique et fantasmes» :

Il restait toujours de ces explications un vide qu'on ne savait comment combler, une zone obscure entre la cause et l'effet : comment arrive-t-on à la page écrite ?

La lune d'où part et revient tout discours et tout poème, tout comme la cour d'Arthur dans les romans arthuriens et le graal lui-même d'où coule tant d'encre, met donc bien en image le lieu de l'inspiration poétique; non pas comme lieu plein de sens, mais comme lieu vide, qui organise tout autour de son centre la constellation des histoires et dans lequel s'opère le passage du chaos de la forêt, espace de l'indistinct et du continu, à l'espace recréé, ordonné et lumineux où les cartes ont enfin la parole.

La fiction de Calvino nous a donc plongés au cœur de l'atelier d'écriture médiéval: présence/absence de l'instance énonciative, entrelacs des récits, combinaisons de motifs narratifs, feuilletage des interprétations qu'ils impliquent, inachèvement, ouvertures du récit vers d'autres continuations possibles, absence et démultiplication de la figure de l'auteur... À son tour, cette mise en image de l'atelier d'écriture médiéval éclaire «la machine littérature» de Calvino. À travers elle, Calvino abat les cartes d'une œuvre littéraire idéale: une oeuvre ouverte, aux entrées multiples, qui pourrait se lire dans tous les sens et dans laquelle l'écrivain se perdrait lui-même comme auteur, se libérerait de sa propre histoire devenue celle des autres, lecteurs et auteurs à leur tour et tout à la fois. Dans les cartes de Calvino, l'auteur prend la figure médiévale du Bateleur. De cette carte, Calvino dit :

Le tarot numéro un est le seul à représenter honnêtement ce que j'ai réussi à être : un prestidigitateur ou illusionniste qui dispose sur son étalage de foire un certain

${ }^{56}$ Le Château, p. 45. 
nombre de figures et qui, les déplaçant, les réunissant, les échangeant, obtient un certain nombre d'effets ${ }^{57}$.

L'étalage du bateleur ressemble à celui de l'écrivain/ poète, que l'on a vu, appliqué, sur la lune de Calvino, à «sa machine à tisser les rimes et les strophes » à la façon des Parques filant, sur la lune de l'Arioste, avec leurs fuseaux, le fil des destinées humaines. Le lecteur est celui même qui, dénouant l'entrelacs de ces fils, reprend la parole.

Dominique Demartini

Université de Paris III-Sorbonne Nouvelle

${ }^{57}$ La Taverne, p. 115. 